\title{
Graves' disease and slipped capital femoral epiphysis: a rare association
}

\begin{abstract}
Introduction: Slipped capital femoral epiphysis (SCFE) is rare and its co-existence with Graves' disease in an individual is even rarer. The aim of this report is to create further awareness among physicians on this association and present a short review of the literature.

Case presentation: We report on a 12-year-old tall, thin, rapidly growing adolescent Nigerian boy with Graves' disease associated with SCFE and whose diagnosis was initially missed. The patient had three medical consultations before the correct diagnosis was made, probably because of the rarity of the association between the two clinical conditions.

Conclusion: SCFE should always be considered in any adolescent presenting with limping and/or hip pain, irrespective of the weight status of the patient, particularly in the presence of an endocrine disorder.
\end{abstract}

Keywords: adolescence, hyperthyroidism, graves' disease, slipped capital femoral epiphysis
Special Issue - 2018

\author{
Alphonsus N. Onyiriuka \\ Endocrine and Metabolic Unit, Department of Child Health, \\ University of Benin Teaching Hospital, Nigeria
}

\begin{abstract}
Correspondence: Alphonsus N. Onyiriuka, Endocrine and Metabolic Unit, Department of Child Health, University of Benin Teaching Hospital, Nigeria, Email alpndiony@yahoo.com
\end{abstract}

Received: March 27, 2017 | Published: November 15, 2018

\section{Introduction}

Slipped capital femoral epiphysis (SCFE) which is also called adolescent coxa vara is the most common adolescent hip disorder. SCFE refers to a posterior and inferior slippage of the proximal femoral epiphysis (femoral head) on the metaphysis (femoral neck), occurring through the epiphyseal growth plate during the early adolescent growth spurt. ${ }^{1}$ Several classifications of SCFE exist but the stability classification is the most useful in terms of predicting prognosis regarding subsequent a vascular necrosis and establishing a treatment plan. ${ }^{2}$ In this classification system, a child is considered to have 'stable' SCFE if the child is able to walk with or without clutches. Where the child is unable to walk with or without clutches, the child is considered to have 'unstable' SCFE. Although the aetiopathogenesis of SCFE remains poorly understood, it is believed to involve mechanical, growth, endocrine and metabolic factors. ${ }^{2}$ In this regard, the plane of cleavage in most SCFEs occurs through the hypertrophic zone of the growth plate. ${ }^{2}$ The pathogenetic mechanisms include factors that either reduces the resistance to shear forces or that increase the stresses across the proximal femoral epiphysis. ${ }^{3}$ Endocrine and metabolic factors are capable of weakening the growth plate but the cellular mechanisms involved are not known with certainty. ${ }^{4}$ Witbreuk et al, after reviewing 56 papers on the subject, concluded that SCFE is most likely the result of a multi factorial event during adolescence when height and weight increase dramatically coupled with disturbances of the delicate hormonal equilibrium secondary to the changing hormonal levels characteristic of adolescence. ${ }^{4}$

The typical cases of SCFE are known to be strongly related to overweight and obesity, accounting for 68 to $81.1 \%$ of this clinical condition. ${ }^{5,6}$ The other varieties of SCFE are atypical and are associated with various pediatric endocrine and metabolic disorders as well as chronic diseases. ${ }^{7}$ Loader et al reviewed 85 cases in which endocrine disorders were associated with SCFE and found that $40 \%$ had hypothyroidism, $25 \%$ had growth hormone deficiency and the remaining $35 \%$ had other conditions such as panhypopituitarism and hyperparathyroidism. ${ }^{8}$ In that report, 5.2 to $6.9 \%$ of patients with SCFE were associated with endocrine disorders. Wells et al reviewed 131 patients with SCFE and found that $7 \%$ of cases were associated with endocrine disorder. ${ }^{9}$ In literature, other recognized causes of atypical SCFE include growth hormone therapy, renal osteodystrophy, vitamin D deficiency, irradiation therapy, Down syndrome and Klinefelter mosaic state. ${ }^{7-11}$ The association between SCFE and overweight/ obesity is better recognized than its association with various endocrine disorders. Therefore, in clinical practice, the latter association is more likely to be overlooked, ${ }^{5}$ resulting in missed/delayed diagnosis. In this regard, Green et al in USA reviewed 102 patients with SCFE and found that this diagnosis was delayed/ missed in $69 \%$ of cases after three visits to primary care physicians. ${ }^{8}$ According to literature, ${ }^{9,11}$ some epidemiologic characteristics of SCFE have been documented. Such characteristics include that SCFE develops shortly before or during accelerated growth and onset of puberty. The ratio of males to females affected is about 2 to $4: 1$. Blacks appear to be more susceptible than whites. Unilateral cases occur three times more frequently than bilateral cases. The left side is involved twice as often as the right in boys, whereas in girls both sides are equally affected. An extensive search of the literature showed that the association between Graves' disease and SCFE is rarer than its association with hypothyroidism. This view is supported by the fact that the association between Graves' disease and SCFE is not mentioned in standard textbooks of pediatric endocrinology ${ }^{12}$ and pediatric orthopedics. ${ }^{11,13}$ In addition, three separate reports on the association of SCFE and endocrine disorders did not include hyperthyroidism (Graves' disease), reflecting the rarity of this association..$^{7,8,10}$ It has been documented that even though hypothyroidism is the most common endocrine abnormality associated with SCFE, its incidence is quite low ${ }^{14}$ and by extension that of hyperthyroidism (Graves' disease) is even lower. The purpose of the present report is to increase the awareness of physicians on the association between Graves' disease and SCFE with a short review of the literature. 


\section{Case report}

We report a case of a 12-year-old Nigerian boy who was referred from a secondary healthcare hospital. He has been on follow-up for Graves' disease for 2 years and was placed on carbimazole and propanolol. The presenting complaints were protrusion of the eyeballs and painless anterior neck swelling, both of 2 years duration. There was a positive history of increased appetite and sweatiness. The patient developed testicular enlargement and pubic hair at the appropriate age. Mother noticed that the child has been growing relatively fast in the past 18 months such that he has become taller than his peers. About 5 months ago, the patient developed left hip pain and limping. The pain was radiating to the thigh. Both the hip pain and limping persisted till presentation in our hospital. The pain is aggravated by standing up from the seated position, walking, bending forward at the waist, and lying on the left side. No history of fever or trauma. There was a negative family history of hip disorder. Since onset of left hip pain, he has had two medical consultations in the secondary healthcare hospital before referral to the pediatric surgeon in our hospital because of no significant regression of the neck swelling. Subsequently, he was referred to the pediatric endocrinologist. Further evaluation in pediatrics endocrinology unit revealed the presence of SCFE as the cause of the left hip disorder, warranting referral to the orthopedic surgeon for urgent surgery to prevent further slippage of the femoral epiphysis and the attendant complications.

Physical examination revealed an adolescent boy with tall stature, exophthalmoses (Figure 1), anterior neck swelling. The swelling was diffuse, smooth, non-tender and moves with deglutition. Anthropometric measurements were weight $43.5 \mathrm{~kg}\left(50^{\text {th }}\right.$ percentile $)$, height $173 \mathrm{~cm}\left(>97^{\text {th }}\right.$ percentile $)$ and body mass index $14.4 \mathrm{~kg} / \mathrm{m}^{2}\left(<5^{\text {th }}\right.$ percentile). His pulse rate was $72 /$ minute, regular and normal volume, blood pressure was $90 / 60 \mathrm{mmHg}$ and the heart sounds were normal without cardiac murmur. Examination of the left hip joint showed limited range of motion, particularly internal rotation. The right hip joint examination was unremarkable. He had an antalgic gait. His sexual maturity rating was Tanner stage 3 . The radiographic findings

Table I Summary of laboratory findings at presentation in our hospital

\begin{tabular}{lll}
\hline Laboratory parameters & Results & Comments \\
\hline Triiodothyronine (T3); mmol/L & $7.42^{*}$ & Elevated (Ref interval 0.90-2.33) \\
Thyroxin (T4); $\mathrm{Hg} / \mathrm{dl}$ & $13.6^{*}$ & Elevated (Ref interval 0.90-2.33) \\
Thyroid stimulating hormone (TSH); $\mu \mathrm{lU} / \mathrm{ml}$ & $0.0^{*}$ & Undetectable (Ref interval 0.5-4.6) \\
Blood glucose; $\mathrm{mmol} / \mathrm{L}$ & 4.8 & Normoglycaemia \\
Packed cell volume; \% & 30 & Low normal \\
Hemoglobin phenotype & $\mathrm{AA}$ & No haemoglobinopathy \\
White blood cell count; $\mathrm{mm}^{-3}$ & 4,600 & Within normal limits \\
Erythrocyte sedimentation rate (Wester green); $\mathrm{mm} / \mathrm{hr}$ & 20 & Elevated \\
Platelet count; $\mathrm{mm}{ }^{-3}$ & 396,000 & Within normal limits \\
Blood film & & Normal red blood cells seen \\
Urinalysis & & No abnormality detected \\
\hline
\end{tabular}

After two years on carbamizole and propanolol: A diagnosis of hyperthyroidism due to Graves' disease associated slipped capital femoral epiphysis was made. The patient was referred to the orthopedic surgeon for urgent surgery to prevent the risk of further slippage of the femoral epiphysis and hip deformities. The anti-thyroid medication was continued. He is being followed up in the clinics in our hospital. confirmed left-sided slipped capital femoral epiphysis (Figure 2). Thyroid scan showed moderate enlargement of both lobes of the thyroid gland, with right slightly larger than the left. The thyroid lobes measured $2.1 \times 5.1 \times 2.1$ and $2.2 \times 3.2 \times 1.6 \mathrm{~cm}$, for right and left lobes, respectively. The parenchyma showed normal architectural and echo pattern with regularity of the outline. There was no evidence of invasion of adjacent blood vessels. The other laboratory findings are summarized in Table 1.

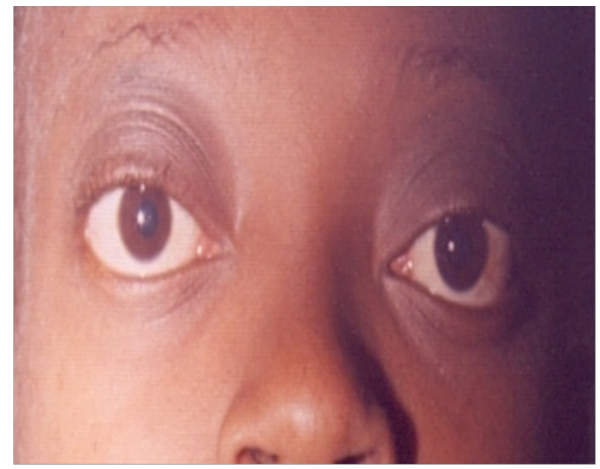

Figure I Exophthalmoses in graves' disease.

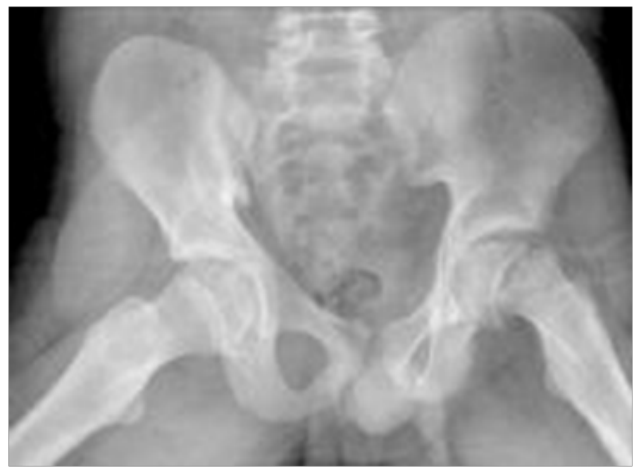

Figure 2 Radiograph showing left SCFE.

\section{Discussion}

The diagnosis of Graves' disease was based on typical clinical features (goiter, exophthalmoses) and elevated thyroid hormones (T3 and T4) and undetectable TSH. The diagnosis of slipped capital femoral epiphysis (SCFE) was suspected based on history of hip 
pain and limping in a tall, thin, pubertal adolescent boy and was subsequently confirmed by the radiographic findings. There was no radiographic evidence of fracture of the neck of left femur, thereby making fracture of the neck of the femur unlikely. Epidemiologically, Legg-Calve-Perthes' disease (a vascular necrosis of the femoral head) is more common than SCFE but the typical age of presentation is 4 to 8 years. ${ }^{1}$ Our patient's age (12 years) was outside this range. More importantly, the radiographic findings in our patient did not support Legg-Calve-Perthes' disease. According to the report by Key ${ }_{1}^{15}$ the chief differential points between SCFE and Legg-Calve-Perthes' disease are age of the patient and the radiographic appearance.

The pathway to diagnosis of SCFE in our patient is instructive and needs consideration. In summary, the patient had three medical consultations (two in the secondary healthcare hospital and one in the tertiary healthcare hospital) before the correct diagnosis of Graves disease associated with SCFE was made. This amounts to a missed/ delayed diagnosis. Such clinical scenario of missed/delayed diagnosis have been severally reported, even in countries with advanced healthcare systems, ${ }^{1,5,7,16,17}$ suggesting the need for greater alertness among physicians with regard to SCFE. The absence of overweight/ obesity in our patient may have contributed to the delay in diagnosis. This view is supported by the report of other investigators. ${ }^{7}$ The practical lesson to be learned is that in adolescents with limping and/ or hip, thigh pain, whether obese or not, SCFE should be considered in the differential diagnosis of the hip disorder. In this way, missed/ delayed diagnosis with the attendant complications will be prevented. In a review by Key, ${ }^{15}$ he stated that the reason why many cases are missed is that the possibility of SCFE is never considered. In that report, he concluded that once the diagnosis of SCFE is considered, the clinical features are usually characteristic. Several reports have emphasized the importance of early diagnosis and appropriate treatment for improved prognosis. ${ }^{18,19}$

In the literature, reports of endocrine disorders associated with SCFE exist with hypothyroidism predominating. ${ }^{1}$ Other endocrine disorders that have been associated with SCFE include growth hormone deficiency, hyperparathyroidism, panhypopituitarism, hypogonadism, gigantism and renal osteodystrophy. ${ }^{7-11}$ In sharp contrast, there is hardly any case report of the association of Graves' disease with SCFE. The mechanisms by which endocrine disorders cause SCFE are poorly understood. In general, it is agreed that SCFE is a disorder occurring during puberty, where the increasing biomechanical load due to accelerated growth (pubertal growth spurt) in conjunction with a relative weakening of the growth plate leads to a displacement of the epiphysis from the metaphysic. ${ }^{6}$ Several reasons have been postulated to explain the weakened growth plate. During normal puberty there is accelerated growth and the growth plate becomes more vertically oriented, resulting in conversion of mechanical forces from compression into shear forces. ${ }^{2}$ In addition, the hypertrophic zone becomes elongated in pubertal adolescents due to high levels of circulating hormones. The resultant widening of the growth plate decreases the threshold for mechanical failure and slip. ${ }^{2}$ Hyperthyroidism is known to cause an increase in growth rate. ${ }^{12}$ With regard to our patient, rapid growth due to pubertal growth spurt coupled with the contribution to growth by hyperthyroidism due to Graves' disease may have jointly weakened the physis (growth plate), resulting in SCFE. The study by Aronson et al, demonstrated that physeal strength is decreased by testosterone whereas it is increased by oestrogen, ${ }^{10}$ accounting for the higher frequency of SCFE in males. The index patient is a boy, therefore testosterone may also have contributed to decreasing physeal strength, leading to slippage of the femoral epiphysis.
The clinical characteristics of the index case need to be considered. Our patient was 12 years old and this corresponds to early adolescent stage. SCFE is known to affect children in early adolescent stage and typically, $10-15$ years of age. ${ }^{1}$ In boys, the mean age of presentation is $13.5 \pm 1.7$ years $^{1}$ and this agrees the age of presentation in our patient. The duration of symptoms (limping and hip pain for 3 months) in our patient classified him as having chronic SCFE. ${ }^{1}$ Chronic slips account $80 \%$ of all cases of SCFE. ${ }^{11}$ Based on stability classification, the index case had stable SCFE. This finding is not surprising as $90 \%$ of cases of SCFE are known to have stable SCFE. ${ }^{1}$ Although our patient's weight status $\left(\mathrm{BMI}<5^{\text {th }}\right.$ percentile for age and sex) was low, his height was greater than the $97^{\text {th }}$ percentile for age and sex. This finding is in agreement with the results of several reports. ${ }^{89}$ The body shape of our patient can be described as thin and tall - a factor commonly present in cases of SCFE associated with endocrinopathies. ${ }^{7-9}$ It appears that height is more strongly related to SCFE associated with endocrine disorders than it is related to SCFE associated with overweight/obesity. This view is supported by the findings of another study. ${ }^{20}$ In that study, the authors stated that height might be useful in distinguishing between typical (idiopathic) and atypical SCFE. In conclusion, SCFE should always be considered in the differential diagnosis of any adolescent presenting with limping and/or hip pain, irrespective of the weight status of the patient, particularly in the presence of an endocrine disorder.

\section{Acknowledgements}

None.

\section{Conflict of interest}

The author declares no conflict of interest.

\section{References}

1. Loder RT. Slipped capital femoral epiphysis. Am Fam Physician. 1998;57(9):2135-2142.

2. Sankar WN, David Horn B, Wells L, et al. Slipped capital femoral epiphysis. Kliegman RM, et al, editors. Nelson Textbook of Pediatrics. 19th edition, Philadelphia: Elsevier Saunders; 2011. p. 2363-2365.

3. Fishkin Z, Amstrong DG, Shah H, et al. Proximal femoral physis shear in slipped femoral capital epiphysis--a finite study. $J$ Pediatr Orthop. 2006;26(3):291-294.

4. Witbreuk M, van Kemenade FJ, van der Sluijs JA, et al. Slipped capital femoral epiphysis and its association with endocrine, metabolic and chronic diseases: a systematic review of the literature. J Child Orthop. 2013;7(3):213-223.

5. Green DW, Reynold RAK, Khan SN, et al. The delay in diagnosis of slipped capital femoral epiphysis: A review of 102 patients. HSS J. 2005;1(1):103-106.

6. Manoff EM, Bunffy MB, Winell JJ. Relationship between body mass index and slipped capital femoral epiphysis. J Pediatr Orthop. 2005;25(6):744-746.

7. Whyte N, Sullivan C. Slipped capital femoral epiphysis in atypical patient. Pediatr Ann. 2016;45(4):e128-134.

8. Loder RT, Wittenberg B, De Silva G. Slipped capital femoral epiphysis associated with endocrine disorders. J Pediatr Orthop. 1995;15(3):349 356.

9. Wells D, King JD, Roe TF, et al. Review of slipped capital femoral epiphysis associated with endocrine disorders. J Pediatr Orthop. 1993;13(5):610-614 
10. Aronson DD, Loder RT, Breur GJ, et al. Slipped capital femoral epiphysis: Current concepts. J Am Acad Orthop Surg. 2006;14(12);666-679.

11. Chung SMK. Handbook of Pediatric Orthopedics. New York, Van Nestrand Reinhold Company Inc; 1996. p. 129-149.

12. Fisher DA, Grueters A. Thyroid disorders in childhood and adolescence. In: Sperlings MA editor. Pediatric Endocrinology. 3rd edition Philadelphia: Saunders Elsevier; 2008: p. 227-253.

13. Lloyd-Roberts GC, Fixsen JA. Orthopaedics in Infancy and Childhood. 2nd edition. London: Butterworth-Heinemann Publishers; 1990: p. 150152 .

14. Richards BS. Slipped capital femoral epiphysis. Pediatr Rev. 1996;17(2):69-70.

15. Key JA. Epiphyseal coxa vara or displacement of the capital epiphysis of the femur in adolescence. Clin Orthop Relat Res. 2013;471(7):20872117.
16. Emary P. Slipped capital femoral epiphysis (SCFE) detected in the chiropractic office: A case report. J Can Chiropr Assoc. 2009;53(3):158164.

17. Kocher MS, Bishop JA, Weed B, et al. Delay in diagnosis of slipped capital femoral epiphysis. Pediatrics. 2004;113(4):e322-325.

18. Rahme D, Comley A, Foster B, et al. Consequences of diagnostic delays in slipped capital femoral epiphysis. J Pediatr Orthop. 2006;15(2):9397.

19. Katz DA. Slipped capital femoral epiphysis: the importance of early diagnosis. Pediatr Ann. 2006;35(2):102-111.

20. Loder RT, Skopelja EN. The epidemiology and demographics of slipped capital femoral epiphysis. ISRN Orthop; 2011. p. 486512. 